\title{
Fractality, Self-Similarity and Complex Dimensions
}

\author{
Michel L. Lapidus and Machiel van Frankenhuijsen
}

To Benoit Mandelbrot, on the occasion of his jubilee.

\begin{abstract}
We present an overview of a theory of complex dimensions of selfsimilar fractal strings, and compare this theory to the theory of varieties over a finite field from the geometric and the dynamical point of view. Then we combine the several strands to discuss a possible approach to establishing a cohomological interpretation of the complex dimensions.
\end{abstract}

\section{Introduction}

In this paper, we survey some aspects of the theory of complex (fractal) dimensions of fractal strings, as developed by the authors in the recent research monograph Fractal Geometry and Number Theory [Lap-vF2] and pursued in the later papers [Lap-vF3-4]. This theory builds upon earlier work of the first author and his collaborators, including Carl Pomerance, Helmut Maier and Christina He [Lap1-3, 5, LapPo1-2, LapMa, HeLap]. In the present paper, we stress the important case of self-similar strings (which are one-dimensional self-similar geometries) along with the geometric and dynamical motivations and applications. For the spectral theory, in particular for a discussion of the question (à la Mark Kac [Kac]) Can one hear the shape of a fractal drum?, going back to the Weyl-Berry Conjecture [Wey1, Ber], we refer the interested reader to the above works and the relevant references therein, including [BroCa, KigLap1-2, Lap4, 6]; see also [Te].

For pedagogical reasons, our goal in $\S \S 2-6$ is to illustrate the mathematical theory of complex dimensions of self-similar strings by means of a few representative examples and results, along with a discussion of some extensions and open problems. We do not aim for the greatest generality or the most complete statements. The interested reader may wish to consult [Lap-vF2-4] (and the relevant references therein) for more details and generality. In $\S 7$ and $\S 8$, as well as in $\S 1.1$ below,

2000 Mathematics Subject Classification. Primary 11M41, 28A80; Secondary 11G20, 11J70.

Key words and phrases. Self-similar fractal strings; lattice vs. nonlattice strings; geometric and dynamical zeta functions; periodicity vs. quasiperiodicity; complex dimensions; Minkowski dimension and measurability; Diophantine approximation; dimension-free regions; explicit formulas; prime orbit theorem; fractal, finite and arithmetic geometries.

This work was partially supported by the US National Science Foundation under the research grant DMS-0070497 (for M.L.L.). 
we explore and clarify the emerging analogies between self-similar strings (and selfsimilar geometries) on the one hand, and varieties over finite fields and arithmetic geometries on the other hand.

We give an overview of the contents of this article. In $\S 2$, we define the notion of fractal string, and introduce the geometric zeta function and (fractal) complex dimensions of a fractal string. In $\S 3$, we focus on self-similar fractal strings. The geometric zeta function of a fractal string $\mathcal{L}$ with lengths $l_{1}, l_{2}, \ldots$ is defined in Equation (2.1). The poles of this function are called the complex dimensions of $\mathcal{L}$. For a string that is self-similar under $N$ scalings by factors $r_{1}, \ldots, r_{N}$ (without gaps, see $\S 3)$, the complex dimensions are the solutions to the equation $r_{1}^{\omega}+\cdots+r_{N}^{\omega}=1$ (see (3.1) and (3.2) below). If $r_{j}=r^{k_{j}}$ for some positive number $r$ and certain positive integers $k_{j}(j=1, \ldots, N)$, this reduces to a polynomial equation, and it is a transcendental equation when such integers do not exist. We introduce the dichotomy of lattice vs. nonlattice self-similar strings in $\S 4$ to make this distinction. This distinction is much like that between rational and irrational real numbers.

The complex dimensions of a lattice string lie periodically on finitely many vertical lines $\operatorname{Re} s=$ constant, separated by a fixed distance $\mathbf{p}=2 \pi / \log r^{-1}$, the oscillatory period of the lattice string (see $\S 4$ ). Therefore, we view a lattice string as an analogue of a variety over a finite field, as explained below. The space of lattice strings is dense in the space of all self-similar strings, and nonlattice strings are shown to have properties that compel us to regard them as analogues of 'infinite dimensional varieties'. Moreover, nonlattice strings with a very large number of scaling ratios form the bridge between self-similar and arithmetic geometries. Some evidence (partly conjectural) for this is presented in $\S 5$. In that section, we apply techniques from Diophantine approximation to obtain 'dimension-free' regions for nonlattice strings. The size of these dimension-free regions depends on how well the ratios $\log r_{j} / \log r_{1}(j=2, \ldots, N)$ can be approximated by rational numbers. In our theory, we deduce from the results in $\S 5$ combined with our 'explicit formulas' from [Lap-vF2] suitable asymptotic expansions with good error term for various geometric $(\S 4)$ or dynamical $(\S 6)$ quantities associated with self-similar fractal strings: the larger the dimension-free region, the better the error term.

In $\S 6$, we explain how the logarithmic derivative of the geometric zeta function of a fractal string can be obtained as the generating function of the periodic orbits of a continuous-time self-similar dynamical system. We thus find an Euler-type product for the geometric zeta function. This is analogous to the discrete-time dynamical system on a variety over a finite field, namely, the Frobenius flow, as explained below. Conjecturally [De, Har2], the corresponding arithmetic dynamical system is the shift on the real line (with infinitesimal generator the derivative). In $\S 7$, we propose an analysis of self-similar strings by measuring the overlap under shifts. Finally, in $\S 8$ we present three tables, summarizing three aspects of these analogies.

As was alluded to above, whereas the work described in $\S \S 2-6$ is completely rigorous and is part of a well-developed (and still growing) mathematical theory of complex dimensions of fractal strings, the content of $\S \S 7-8$ is of a more speculative nature and should, in the long-term, be the object of further investigations and developments. It builds upon well-known analogies in arithmetic (or Diophantine) geometry (see, e.g., [Wei1, Kat, ParsSh, De]) and upon new ones proposed 
in [Lap-vF2, §10.5] and in the first author's forthcoming book [Lap8], In Search of the Riemann Zeros.

1.1. Self-Similar and Finite Geometries. We close this introduction by providing some motivations and background material on varieties over a finite field. We want to reassure the reader mainly interested in fractal geometry that no prior knowledge of arithmetic or algebraic geometry is required to read this paper.

The analogy between the geometric zeta function of self-similar fractal strings and the zeta function of a variety over a finite field becomes apparent in the following simplest example. The zeta function of the affine line ${ }^{1}$ over $\mathbb{F}_{p}$ is defined as $\zeta_{\mathbb{A}^{1} / \mathbb{F}_{p}}(s)=\left(1-p \cdot p^{-s}\right)^{-1}$. We compare this with the geometric zeta function of the Cantor string, to be introduced in Example 3.2,

$$
\zeta_{\mathrm{CS}}(s)=\frac{1}{1-2 \cdot 3^{-s}} .
$$

Writing $D=\log _{3} 2=\log 2 / \log 3$ for the Minkowski dimension of the Cantor string, as will be explained in $\S 2$, we find $\zeta_{\mathbb{A}^{1} / \mathbb{F}_{3}}(s)=\zeta_{\mathrm{CS}}(s+D-1)$ or $\zeta_{\mathbb{A}^{1} / \mathbb{F}_{2}}(s)=\zeta_{\mathrm{CS}}(s D)$. These zeta functions have one line of poles, at $\omega=1+i k \mathbf{p}(k \in \mathbb{Z}$ and $\mathbf{p}=2 \pi / \log 2$ or $\mathbf{p}=2 \pi / \log 3$, respectively).

In general, one defines the zeta function of a variety over a finite field as the generating function (or Mellin transform) of the counting measure of the positive divisors on the variety. As such, it is immediately clear that the zeta function can be obtained as an Euler product of factors that are defined in terms of the prime divisors of the variety. A variety over a finite field comes equipped with an action of the Frobenius endomorphism. ${ }^{2}$ This defines a discrete-time flow on the variety, the orbits of which are conjugacy classes of points on the variety, which (for curves) are the prime divisors of the variety. The logarithmic derivative of the Euler product is the generating function of the counting measure of the orbits of Frobenius.

One of the most important developments in the theory of algebraic varieties was the definition and subsequent development of a cohomology theory. Indeed, the so-called étale cohomology, which captures the combinatorics of families of étale covers of the variety, provides a theory that can be compared to the classical singular homology and cohomology theories of manifolds. Since the étale theory is defined purely algebraically, it allows application to varieties defined over a finite field. In particular, one recovers the zeta function of the variety as the alternating product of the characteristic polynomials of the induced action of Frobenius on the étale cohomology groups. The poles of this zeta function are located on 'integer vertical lines' Re $s=0,1, \ldots, n$, where $n$ is the dimension of the variety, and the zeros are located on 'half-integer vertical lines' $\operatorname{Re} s=\frac{1}{2}, \frac{3}{2}, \ldots, n-\frac{1}{2}$. (See, e.g., [Kat, FreKie, De, Wei1-2, 4, ParsSh,I] for further information about this beautiful subject.)

This theory was first modelled on the theory of the Riemann zeta function, which is the first example of the zeta function of an 'arithmetic geometry', namely, the spectrum of $\mathbb{Z}$. There exist only one-dimensional ${ }^{3}$ arithmetic geometries. The (completed) Riemann zeta function has simple poles at $s=1$ and $s=0$, hence one

\footnotetext{
${ }^{1}$ Here, and henceforth, $p$ is a prime number and $\mathbb{F}_{p}$ denotes the finite field with $p$ elements.

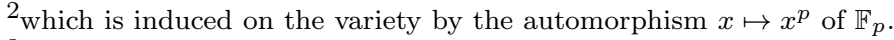

${ }^{3}$ Indeed, the product of the spectrum of $\mathbb{Z}$ with itself has coordinate ring $\mathbb{Z} \otimes \mathbb{Z}=\mathbb{Z}$, hence the product reduces to the diagonal [Har1].
} 
could say (by analogy with the case of a curve over a finite field) that the cohomology groups $H^{0}$ and $H^{2}$ are one-dimensional; i.e., the spectrum of $\mathbb{Z}$ (completed at the archimedean place) is connected and one-dimensional. On the other hand, if the Riemann hypothesis holds, then the middle cohomology group $H^{1}$ could possibly be defined, and should be infinite dimensional. Moreover, the logarithmic derivative of the Riemann zeta function is the generating function of the primepower counting function. It is not known how the prime numbers $p$ can be viewed as the (primitive) periodic orbits (of length $\log p$ ) of a flow, but there is some indication that such a flow, if it exists, should take into account the smooth (archimedean) structure [De,Har2]. The simplest such flow is the shift on the real line.

REMARK 1.1. In a book in preparation ([Lap8], announced in [Lap7]), the first author has proposed a suitable notion of 'quantized fractal string', coined 'fractal membrane', and has significantly extended the above analogy between self-similar geometries and arithmetic geometries. In particular, lattice strings (or membranes) correspond to varieties over finite fields whereas nonlattice strings (or membranes) are viewed as a counterpart of number fields. ${ }^{4}$ Moreover, the scaling ratios of self-similar geometries (or of 'self-similar membranes' [Lap8]) play the role of the 'generalized primes' attached to fractal membranes, while expressions like (3.1) for the geometric zeta function of a self-similar string (with possibly infinitely many scaling ratios and gaps) correspond to the Euler product representation (of the same nature as that for the classical Riemann zeta function [In, Pat, Ti]) of the partition function of a fractal membrane (as obtained in [Lap8]). Finally, in joint work of the first author with Ryszard Nest [LapNe], it was recently shown that the fractal membranes (resp. self-similar membranes) introduced in [Lap8] can be rigorously constructed as the second quantization of fractal strings by using FermiDirac (resp., Gibbs-Boltzmann or 'free') statistics along with aspects of the theory of operator algebras and of Connes' noncommutative geometry [Con].

\section{Fractal Strings}

A fractal string is a one-dimensional drum with fractal boundary. Thus, a fractal string is a bounded open subset $\mathcal{L}$ of the real line. We assume here that the connected components "touch" each other; i.e., the closure of $\mathcal{L}$ is a bounded connected interval $I$, and the boundary $\partial \mathcal{L}$ of $\mathcal{L}$ is a disconnected fractal subset of $\mathbb{R}$ with measure zero. The lengths of the connected components (i.e., open intervals) of the string are called the lengths of $\mathcal{L}$ (a better terminology would be to speak of the strings of a fractal harp; see [Lap-vF2, p. 8]). We can list the lengths of a fractal string in nonincreasing order

$$
\mathcal{L}: l_{1} \geq l_{2} \geq l_{3} \geq \ldots
$$

each repeated according to its multiplicity. The geometric zeta function of the fractal string is determined by the sequence $\mathcal{L}$ :

$$
\zeta_{\mathcal{L}}(s)=\sum_{j=1}^{\infty} l_{j}^{s} .
$$

\footnotetext{
${ }^{4}$ finite extensions of the field of rational numbers; see, e.g., [ParsSh, I \& II].

${ }^{5}$ We might say, the fractal string is irreducible or connected, in the sense of irreducible schemes or connected manifolds.
} 
Thus, for example, $\zeta_{\mathcal{L}}(1)=|I|$ is the length of the closure of the fractal string in the real line. On the other hand, $\zeta_{\mathcal{L}}(0)$ is the number of lengths of $\mathcal{L}$, and we exclude the case when this number is finite. Recall that the abscissa of convergence of a Dirichlet series is defined as the smallest real number $\sigma_{c}$ such that the series (2.1) converges (absolutely) in the half-plane $\operatorname{Re} s>\sigma_{c}$. Thus the abscissa of convergence of $\zeta_{\mathcal{L}}$ satisfies $0 \leq \sigma_{c} \leq 1$.

We now compute the Minkowski (or box) dimension of the complement of the string in its interval. (See also [Lap1-2,LapPo1].) When the two endpoints of an interval of length $l_{j}$ are covered by intervals (or disks) of radius $\varepsilon$, then these disks overlap if $l_{j}<2 \varepsilon$, covering a length $l_{j}$, or they do not overlap if $l_{j} \geq 2 \varepsilon$, in which case they cover a length of $2 \varepsilon$. Moreover, there are the two pieces of length $\varepsilon$ sticking out to the left and the right of $I$. Thus the length $V(\varepsilon)=V_{\mathcal{L}}(\varepsilon)$ of the (two-sided) $\varepsilon$-neighborhood of the string is given by ${ }^{6}$

$$
V(\varepsilon)=|\partial \mathcal{L}+(-\varepsilon, \varepsilon)|=2 \varepsilon+\sum_{j: l_{j} \geq 2 \varepsilon} 2 \varepsilon+\sum_{j: l_{j}<2 \varepsilon} l_{j} .
$$

REMARK 2.1. In [Lap-vF1-4], we used the inner neighborhood of the string, to allow for strings with lengths not necessarily embedded in the real line. The present approach is less intrinsic, but more suitable to study properties of self-similarity (also see the end of $\S 2.1$ ).

The Minkowski dimension $D$ of $\partial \mathcal{L}$ is the infimum of the numbers $d$ such that $V(\varepsilon)=o\left(\varepsilon^{1-d}\right)$ as $\varepsilon \rightarrow 0^{+}: D=\inf \left\{d \geq 0: \varepsilon^{d-1} V(\varepsilon) \rightarrow 0\right.$ as $\left.\varepsilon \rightarrow 0^{+}\right\}$. Further, $\partial \mathcal{L}$ is Minkowski measurable if the following limit exists in $(0, \infty)$,

$$
\mathcal{M}=\mathcal{M}(D ; \mathcal{L}):=\lim _{\varepsilon \rightarrow 0^{+}} \varepsilon^{D-1} V(\varepsilon) .
$$

(See Remark 3.1 below and, e.g., [Lap1-3, LapPo1-2, Fa2, Ch. 3, Mat, Ch. 5].)

We now show that the Minkowski dimension can be recovered as the abscissa of convergence of $\zeta_{\mathcal{L}}$. (This was first observed by the first author in [Lap2-3] using a result of Besicovich and Taylor [BesT]; see also [LapPo1,LapMa].)

THEOREM 2.2. The abscissa of convergence $\sigma_{c}$ of the geometric zeta function $\zeta_{\mathcal{L}}$ coincides with $D$, the Minkowski dimension of $\partial \mathcal{L}$.

Proof. Let $s>d>D$. There exists a constant $C_{1}$ such that $V(\varepsilon) \leq C_{1} \varepsilon^{1-d}$. For $\varepsilon=l_{n} / 2$, we obtain $(n+1) l_{n} \leq(n+1) l_{n}+\sum_{j=n+1}^{\infty} l_{j}=V\left(l_{n} / 2\right) \leq C_{1}\left(l_{n} / 2\right)^{1-d}$. It follows that $l_{n}^{s} \leq C_{2}(n+1)^{-s / d}$, for some constant $C_{2}$. Hence the series (2.1) converges for $s>d$, so that $\sigma_{c} \leq d$. Since this holds for every $d>D$, we obtain $\sigma_{c} \leq D$. If $\sigma_{c}=1$, we conclude that $D=\sigma_{c}$, since $V(\varepsilon) \leq 2 \varepsilon+|I|$ is bounded.

Otherwise, let $\sigma_{c}<s<1$. Then the series (2.1) converges. Since the sequence of lengths is nonincreasing, we find that $n l_{n}^{s} \leq \sum_{j=1}^{n} l_{j}^{s} \leq C_{3}$. Hence $l_{n} \leq\left(C_{3} / n\right)^{1 / s}$. Given $\varepsilon>0$, it follows that $l_{n}<2 \varepsilon$ for $n>C_{3}(2 \varepsilon)^{-s}$. For $j \leq C_{3}(2 \varepsilon)^{-s}$, we estimate the $j$-th term (in the first or the second sum) in (2.2) by $2 \varepsilon$, and for $j>C_{3}(2 \varepsilon)^{-s}$, we estimate this term in the second sum by $\left(C_{3} / j\right)^{1 / s}$. Thus we find $V(\varepsilon) \leq C_{4}(2 \varepsilon)^{1-s}$, so that $D \leq s$. Since this holds for every real number $s>\sigma_{c}$, we conclude that $D \leq \sigma_{c}$. Hence these two quantities coincide.

\footnotetext{
${ }^{6}$ For two subsets of $\mathbb{R}, A+B$ denotes the set $\{a+b: a \in A, b \in B\}$. Thus, $A+(-\varepsilon, \varepsilon)$ is the open $\varepsilon$-neighborhood of $A$. Also, $|A|$ denotes Lebesgue measure in $\mathbb{R}$.
} 
2.1. Complex Dimensions of a Fractal String. We assume that $\zeta_{\mathcal{L}}$ has a meromorphic continuation to a neighborhood of the half-plane Re $s \geq D$. Clearly then, $D$ is a pole of $\zeta_{\mathcal{L}}$. The geometric zeta function may have other singularities, which are then located on or to the left of the line $\operatorname{Re} s=D$. These singularities are necessarily poles, and they are called the complex dimensions of $\mathcal{L}$. We denote by $\mathcal{D}=\mathcal{D}_{\mathcal{L}}$ the set of (visible) complex dimensions of $\mathcal{L}$ (within the given region). Clearly, it is a discrete subset of $\mathbb{C}$, and therefore is at most countable.

The complex dimensions enter into the explicit formulas for the various geometric, dynamical, and spectral quantities related to $\mathcal{L}$. These (pointwise or distributional) explicit formulas - in the sense of number theory [In, Pat, Ti, Wei3], but more general - are established in [Lap-vF2, Ch.4] and then applied and adapted to a number of situations throughout the rest of that book, especially in [LapvF2, Chs. 5-7,9] (see also [Lap-vF3-4], along with the forthcoming second edition of [Lap-vF2]).

As an illustration, we have the explicit formula for the volume (i.e., length) of the tubular neighborhood $V(\varepsilon)$, which we formulate here in the case when the complex dimensions are simple. See [Lap-vF2, Ch.6] for the general formulation and additional details.

THEOREM 2.3. Let $\zeta_{\mathcal{L}}(s)$ have a meromorphic continuation to a neighborhood of $\operatorname{Re} s \geq \sigma_{0}$ (for some $\sigma_{0} \leq D$ ), with simple poles and such that $s=0$ is not a pole of $\zeta_{\mathcal{L}}$. Then, under mild growth conditions on $\zeta_{\mathcal{L}}$,

$$
V(\varepsilon)=\sum_{\omega} \operatorname{res}\left(\zeta_{\mathcal{L}} ; \omega\right) \frac{(2 \varepsilon)^{1-\omega}}{\omega(1-\omega)}+2 \varepsilon\left(1+\zeta_{\mathcal{L}}(0)\right)+O\left(\varepsilon^{\sigma_{0}}\right) \text { as } \varepsilon \rightarrow 0^{+} .
$$

The sum extends over all poles of $\zeta_{\mathcal{L}}$ in $\sigma_{0} \leq \operatorname{Re} \omega \leq D$ (i.e., over all 'visible' complex dimensions $\omega$ of $\mathcal{L}$ ).

This formula expresses $V(\varepsilon)$ as a sum of oscillatory terms of the form $C_{\omega} \varepsilon^{1-\omega}$, where $\omega$ runs over all (visible) complex dimensions of $\mathcal{L}$. These terms grow asymptotically as $\varepsilon^{\operatorname{Re} \omega}$ in size, and have a multiplicative period $r=\exp (2 \pi / \operatorname{Im} \omega)$; i.e., $\varepsilon^{\omega}$ and $(r \varepsilon)^{\omega}$ have the same argument. The coefficient of each oscillatory term depends in particular on $\operatorname{res}\left(\zeta_{\mathcal{L}} ; \omega\right)$, the residue of the geometric zeta function at $\omega$. By analogy with Weyl's formula for the volume of the $\varepsilon$-neighborhoods of a (smooth) submanifold of Euclidean space ([Wey2, BeGo, §6.6-6.9, esp. Thm. 6.9.9]), we expect eventually to be able to obtain a suitable geometric interpretation of these values; see $[$ Lap-vF2, $\S 6.1 .1]$. For example, in view of $(2.3)$, the residue at $D$ is closely related to the Minkowski content (see [Lap-vF2, Thm. 6.12]):

$$
\mathcal{M}(D ; \mathcal{L})=\operatorname{res}\left(\zeta_{\mathcal{L}} ; D\right) \frac{2^{1-D}}{D(1-D)}
$$

provided that there are no other complex dimensions with real part $D$. Moreover, this is an important reason why we prefer to work here with two-sided (rather than with one-sided) neighborhoods of $\mathcal{L}$ (see Remark 2.1 above). Indeed, in the former case, the geometric interpretation of Weyl's tube formula [Wey2] is valid for submanifolds of any dimension, whereas in the latter case, it is only valid for even-dimensional submanifolds; see, e.g., [BeGo], loc. cit. 


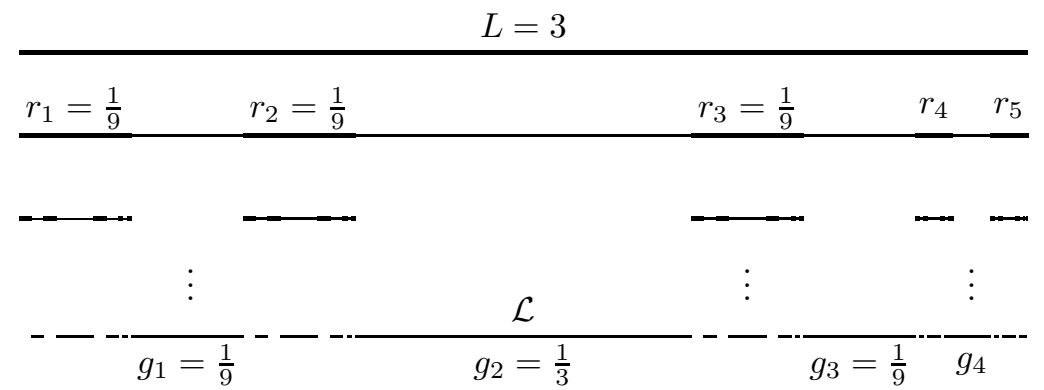

Figure 1. The modified Cantor string, with five scaling ratios $r_{1}=r_{2}=r_{3}=\frac{1}{9}, r_{4}=r_{5}=\frac{1}{27}$, and four gaps $g_{1}=g_{3}=\frac{1}{9}$, $g_{2}=\frac{1}{3}, g_{4}=\frac{1}{27}$.

\section{Self-Similar Fractal Strings}

Let positive scaling ratios $r_{1} \geq r_{2} \geq \cdots \geq r_{N}$ and gaps $g_{1}, \ldots, g_{K}$ be given such that $r_{1}+\cdots+r_{N}+g_{1}+\cdots+g_{K}=1$. Figure 1 explains how one constructs a selfsimilar fractal string with an 'initiator' $r_{1}, \ldots, r_{N}, g_{1}, \ldots, g_{K}$ in an initial interval of length $L$. (See [Fr, Lap-vF4].) The initial interval is replaced by scaled copies, leaving gaps in between. The gaps and scaled gaps form the lengths of the string $\mathcal{L}$, which are the products of the form $L g_{k} r_{j_{1}} \ldots r_{j_{q}}, 1 \leq k \leq K, 1 \leq j_{1}, \ldots, j_{q} \leq N$, $q \geq 0$. The multiplicity of a length $l$ is the number of such products with value $l$. In $[$ Lap-vF2,4], we have computed the geometric zeta function of $\mathcal{L}$,

$$
\zeta_{\mathcal{L}}(s)=\frac{L^{s} \sum_{k=1}^{K} g_{k}^{s}}{1-\sum_{j=1}^{N} r_{j}^{s}} .
$$

It follows that the geometric zeta function of a self-similar string has a meromorphic continuation to the entire complex plane, given by (3.1). Further, the set $\mathcal{D}_{\mathcal{L}}$ of complex dimensions of $\mathcal{L}$ is a subset of the set of solutions of the equation

$$
r_{1}^{\omega}+r_{2}^{\omega}+\cdots+r_{N}^{\omega}=1 \quad \omega \in \mathbb{C} .
$$

It may be a proper subset if zeros of the denominator are cancelled by zeros of the numerator of $\zeta_{\mathcal{L}}$, as we show in this paper (see Examples 3.2 and 3.6 below). Moreover, $D$ is the unique real solution to (3.2). Note that $D$ is always a simple root of this equation, and that it is never cancelled by a root of the numerator of $\zeta_{\mathcal{L}}$, since the numerator of (3.1) does not have real roots.

Of particular interest is the case where there is only one gap, $K=1$, necessarily of size $g=1-r_{1}-\cdots-r_{N}$. Then, $\zeta_{\mathcal{L}}(s)=(g L)^{s} /\left(1-\sum_{j=1}^{N} r_{j}^{s}\right)$, and the complex dimensions are exactly the (complex) solutions of Equation (3.2). This case was studied in [Lap-vF2]; see also $\S 6$. Another natural example is when the scaled copies of $I$ are interspersed by gaps, so that $K=N-1$. Only in this case, $\zeta_{\mathcal{L}}(0)=-1$, so that there is no term of order $\varepsilon$ in $(2.4)$. In general, $\zeta_{\mathcal{L}}(0)=K /(1-N)$.

REMARK 3.1. After a possible rearrangement, any self-similar set in $\mathbb{R}$ (without too much overlap ${ }^{7}$ ) can be viewed as the boundary $\partial \mathcal{L}$ of a self-similar string $\mathcal{L}$ (possibly with multiple gaps); and conversely. Further, it is well-known that for such self-similar sets, the Minkowski and the Hausdorff dimensions coincide. Moreover,

\footnotetext{
7 that is, satisfying the so-called 'open set condition' [Hut, Fa2, p. 112].
} 
since $D$ is the unique real solution of the 'complexified Moran equation' (3.2) [Mor], it also coincides with the similarity dimension of $\partial \mathcal{L}$ (in the sense of Mandelbrot [Man]); see, e.g., [Fa2, Thms. 9.1, 9.3].

We next give the example of the simplest self-similar string. The second example shows that the initiator of a self-similar string is in general not unique.

Example 3.2 (Cantor and Modified Cantor String). The string with five scaling ratios $r_{1}=r_{2}=r_{3}=1 / 9, r_{4}=r_{5}=1 / 27$ and four gaps $g_{1}=1 / 3$, $g_{2}=g_{3}=1 / 9, g_{4}=1 / 27$ in an interval of length 3 (see Figure 1 ), has for geometric zeta function

$$
\zeta_{\mathcal{L}}(s)=3^{s} \frac{3^{-s}+2 \cdot 3^{-2 s}+3^{-3 s}}{1-3 \cdot 3^{-2 s}-2 \cdot 3^{-3 s}}
$$

The denominator factors as follows: $1-3 x^{2}-2 x^{3}=(1-2 x)(1+x)^{2}$, and the numerator as $x(1+x)^{2}$, with $x=3^{-s}$. Hence the zeta function simplifies to the geometric zeta function (1.1) of the Cantor string, with two scaling ratios $r_{1}=$ $r_{2}=1 / 3$ and one gap $g_{1}=1 / 3$, in an interval of length 3 . Thus the sequence of its lengths coincides with the sequence of lengths of the connected components of the complement of the Cantor set, see [Lap-vF2, Ex. 2.2.1]. The poles (of (3.3) or (1.1)) are simple, located at $D+i k \mathbf{p}(k \in \mathbb{Z})$, with $D=\log _{3} 2$ and $\mathbf{p}=2 \pi / \log 3$; the residue at each pole is equal to $1 / \log 3$. In particular, the complex dimensions of the Cantor string lie periodically on a single vertical line, defined by $\operatorname{Re} s=D$.

REMARK 3.3. The initiator of a self-similar fractal string is not unique since one can always find a Dirichlet polynomial $L^{s}\left(g_{1}^{s}+\cdots+g_{K}^{s}\right)$ with positive coefficients such that the product of this Dirichlet polynomial with the denominator of $\zeta_{\mathcal{L}}$ is again of the form $1-r_{1}^{s}-\cdots-r_{N}^{s}$. Indeed, repeated application of the identity $\left(1-r_{1}^{s}-\cdots-r_{N}^{s}\right)^{-1}=\left(1+r_{1}^{s}+\cdots+r_{N}^{s}\right) /\left(1-\left(r_{1}^{s}+\cdots+r_{N}^{s}\right)^{2}\right)$ shows that every fractal string has infinitely many initiators. Example 3.6 below gives an alternative initiator for the Fibonacci string.

The following example shows that the complex dimensions of a self-similar fractal string are not necessarily simple (see [Lap-vF2, Ex. 2.2.3]).

Example 3.4 (Multiple Complex Dimensions). The string with the same scaling ratios as in Example 3.2 but one gap $g_{1}=1 / 3+2 / 9+1 / 27$ has complex dimensions on a line $\pi i / \log 3+2 k \pi i / \log 3(k \in \mathbb{Z})$, each with multiplicity two.

ExAmple 3.5 (Fibonacci String). The Fibonacci string, introduced in [LapvF2, Ex. 2.2.2], has the initiator $r_{1}=1 / 2, r_{2}=g_{1}=1 / 4$, in an interval of length 4 . Thus the geometric zeta function of this string is $\zeta_{\mathrm{Fib}}(s)=\left(1-2^{-s}-2^{-2 s}\right)^{-1}$. This is a string with lengths $2^{-n}$, of multiplicity $f_{n+1}$, the $(n+1)$-st Fibonacci number (defined by $f_{0}=0, f_{1}=1$ and $f_{n+1}=f_{n}+f_{n-1}$ ). We find two (discrete) lines of complex dimensions: $\{D+i n \mathbf{p}\}_{n \in \mathbb{Z}}$, with $D=\log _{2} \phi$ and $\mathbf{p}=2 \pi / \log 2$, and the line $\{-D-i \mathbf{p} / 2+i n \mathbf{p}\}_{n \in \mathbb{Z}}$.

The volume $V_{\mathrm{Fib}}(\varepsilon)$ of the tubular neighborhood of the Fibonacci string can be computed directly, thereby illustrating formula $(2.2)$. Let $\phi=(1+\sqrt{5}) / 2$ be the golden ratio and recall that $f_{n}=\left(\phi^{n}-(1-\phi)^{n}\right) / \sqrt{5}$. By $(2.2)$, we have $V_{\mathrm{Fib}}(\varepsilon)=2 \varepsilon \sum_{2^{-n}>2 \varepsilon} f_{n+1}+\sum_{2^{-n}<2 \varepsilon} f_{n+1} 2^{-n}$. By the above formula for $f_{n}$, both sums are geometric. We write $x=\log _{2}(2 \varepsilon)^{-1}$, so that $x$ increases by one unit if $\varepsilon$ 
is halved in value. Evaluating the above sums, we find that

$$
\begin{aligned}
V_{\mathrm{Fib}}(\varepsilon)= & \frac{(2 \varepsilon)^{1-D}}{\sqrt{5}}\left(\phi^{3} \phi^{-\{x\}}+\phi^{4}(\phi / 2)^{-\{x\}}\right) \\
& +\frac{(2 \varepsilon)^{1+D}}{\sqrt{5}}(-1)^{[x]}\left(\phi^{-3} \phi^{\{x\}}-\phi^{-4}(2 \phi)^{\{x\}}\right) .
\end{aligned}
$$

Here, $[x]$ denotes the integer part, and $\{x\}=x-[x]$ the fractional part of the real number $x=\log _{2}(2 \varepsilon)^{-1}$. The two functions in parentheses are periodic (and continuous). Computing their Fourier series, we recover for the Fibonacci string the explicit formula (2.4), even without error term: ${ }^{8}$

$$
\begin{aligned}
V_{\mathrm{Fib}}(\varepsilon)= & \frac{\phi}{\sqrt{5} \log 2} \sum_{k=-\infty}^{\infty} \frac{(2 \varepsilon)^{1-D-i k \mathbf{p}}}{(D+i k \mathbf{p})(1-D-i k \mathbf{p})} \\
& +\frac{\phi-1}{\sqrt{5} \log 2} \sum_{k=-\infty}^{\infty} \frac{(2 \varepsilon)^{1+D-i \mathbf{p} / 2-i k \mathbf{p}}}{(-D+i \mathbf{p} / 2+i k \mathbf{p})(1+D-i \mathbf{p} / 2-i k \mathbf{p})}
\end{aligned}
$$

ExAmple 3.6 (Modified Fibonacci String). The initiator $r_{1}=r_{2}=g_{1}=1 / 4$, $r_{3}=g_{2}=1 / 8$ in an interval of length 4 generates a string with geometric zeta function $\zeta_{\mathcal{L}}(s)=2^{2 s}\left(2^{-2 s}+2^{-3 s}\right) /\left(1-2 \cdot 2^{-2 s}-2^{-3 s}\right)=\left(1-2^{-s}-2^{-2 s}\right)^{-1}$. Hence the sequence of lengths of this string coincides with that of the Fibonacci string of Example 3.5.

\section{The Lattice and the Nonlattice Case: Periodicity vs. Quasiperiodicity}

The lattice/nonlattice dichotomy comes from renewal theory [Fel, Ch. XI]. It was used in a related context in [Lal1-2,St1-2] and [Lap3]; see also, e.g., [KigLap1, Lap4, Fa3-4, Lap-vF2, HamLap], and the relevant references therein.

4.1. The Lattice Case. The self-similar fractal string $\mathcal{L}$ is a lattice string if the multiplicative subgroup $G=\prod_{j=1}^{N} r_{j}^{\mathbb{Z}}$ of $\mathbb{R}_{+}^{*}$ generated by $r_{1}, \ldots, r_{N}$ has rank 1 as a free abelian group. In that case, $G$ is a discrete subgroup $r^{\mathbb{Z}}$ of $\mathbb{R}_{+}^{*}$. The number $r \in(0,1)$ is called the multiplicative generator of $\mathcal{L}$.

Examples 3.2 and 3.4-3.6 are all examples of lattice strings. As we saw in the example of the Fibonacci string, in the lattice case we can obtain complete information about the geometry of the string. This is reflected in the following theorem, which describes the structure of the complex dimensions of a lattice string (see [Lap-vF2, Thm. 2.13]).

Theorem 4.1 (Lattice Case: Periodic Patterns). Let $\mathcal{L}$ be a lattice self-similar string with multiplicative generator $r$. Then $\zeta_{\mathcal{L}}$ is a rational function of $r^{s}$, and hence there exist finitely many solutions $\omega_{1}:=D, \omega_{2}, \ldots, \omega_{m}$ of Equation (3.2) such that the complex dimensions of $\mathcal{L}$ lie on finitely many vertical lines

$$
\omega=\omega_{u}+i k \mathbf{p}, \quad k \in \mathbb{Z}, \quad u=1, \ldots, m,
$$

where $\mathbf{p}=2 \pi / \log r^{-1}$ is the oscillatory period of $\mathcal{L}$. In particular, the complex dimensions of a lattice string exhibit a periodic pattern.

\footnotetext{
${ }^{8}$ The general theory of $[\mathrm{Lap}-\mathrm{vF} 2, \mathrm{Ch} .4]$ also enables us to obtain this explicit formula without error term. An analogous statement holds for all lattice strings (see $\S 4.1$ and [LapvF2, Thms. 4.8,6.21]).
} 
Since there are complex dimensions other than $D$ with real part equal to $D$, it follows from Equation (2.4) that $\varepsilon^{D-1} V(\varepsilon)$ oscillates without a limit as $\varepsilon \rightarrow 0^{+}$. Hence a lattice string is not Minkowski measurable.

To prove this, observe that in the lattice case, there exist integers $1 \leq k_{1} \leq$ $k_{2} \leq \cdots \leq k_{N}$ such that $r_{j}=r^{k_{j}}$ for $j=1, \ldots, N$, so that Equation (3.2) is equivalent to

$$
z^{k_{1}}+z^{k_{2}}+\cdots+z^{k_{N}}=1, \quad z=r^{\omega} .
$$

Hence $m$, the number of lines of complex dimensions, satisfies $m \leq k_{N}$. Moreover, some complex dimensions, and even entire lines of complex dimensions, can be canceled by roots of the numerator of (3.1). See also Remark 8.1.

For example, if $L=1$ and $\mathcal{L}$ has one gap, then we obtain, for $0<\varepsilon<(2 r)^{-1}$,

$$
\begin{aligned}
V(\varepsilon)= & \operatorname{res}\left(\zeta_{\mathcal{L}}(s) ; D\right) \sum_{n \in \mathbb{Z}} \frac{(2 \varepsilon)^{1-D-i n \mathbf{p}}}{(D+i n \mathbf{p})(1-D-i n \mathbf{p})} \\
& +\sum_{\operatorname{Re} \omega<D} \operatorname{res}\left(\frac{\zeta_{\mathcal{L}}(s)(2 \varepsilon)^{1-s}}{s(1-s)} ; \omega\right)+2 \varepsilon\left(\zeta_{\mathcal{L}}(0)+1\right) \\
= & \operatorname{res}\left(\zeta_{\mathcal{L}}(s) ; D\right)(2 \varepsilon)^{1-D} G\left(\log _{r}(2 \varepsilon)\right)+o\left(\varepsilon^{1-\Theta}\right), \text { as } \varepsilon \rightarrow 0^{+},
\end{aligned}
$$

where the line $\operatorname{Re} s=\Theta<D$ is the first line of complex dimensions that lies (strictly) to the left of $D$, and $G$ is the periodic function

$$
G(x)=\sum_{n \in \mathbb{Z}} \frac{e^{2 \pi i n x}}{(D+i n \mathbf{p})(1-D-i n \mathbf{p})}=\log r^{-1}\left(\frac{r^{D\{x\}}}{1-r^{D}}+\frac{r^{(D-1)\{x\}}}{r^{D-1}-1}\right) .
$$

Since this periodic function is nonconstant, it follows that $\mathcal{L}$ is not Minkowski measurable.

In general, each (discrete) line of complex dimensions $\left\{\omega_{u}+i k \mathbf{p}: k \in \mathbb{Z}\right\}$ (for $u=$ $1, \ldots, m)$ of a lattice string gives rise to a (multiplicatively) periodic function times a suitable power of $\varepsilon$ (if $\omega_{u}$ is a simple pole ${ }^{9}$ ) in the corresponding explicit formula (see [Lap-vF2, §6.3.1, esp. Thm. 6.21, Ex. 6.25]).

The oscillatory period $\mathbf{p}$ of a lattice string $\mathcal{L}$ can be interpreted as the generator of the spectrum of self-similarity of $\mathcal{L}$. Indeed, the lengths of $\mathcal{L}$ are of the form $r^{n} g_{k} L$ $(k=1, \ldots, K, n \in \mathbb{N})$, with a multiplicity that grows like $r^{D n}$; i.e., exponentially with $n$. Figure 2 gives diagrams of the complex dimensions of six lattice strings that approximate the nonlattice string of Example 4.5. For example, the diagram labeled $\sqrt{2} \approx 41 / 29$ gives the complex dimensions of the lattice string with $r_{1}=r^{29}$, $r_{2}=r^{2 \cdot 29}$, and $r_{3}=r^{41+29}$, where $r=2^{-1 / 29}$ (and one gap $g=1 / 4-r_{3}$ ). Its oscillatory period is $\mathbf{p}=58 \pi / \log 2 \approx 262.9$.

The lower parts of the diagrams in this figure give the relative density of the real parts of the complex dimensions within one period. These density graphs were obtained in the following way. For each solution $z$ of Equation (4.2), we computed the real part of the complex dimension $\omega$, namely $\operatorname{Re} \omega=\log _{r}|z|$. Then we ordered this set to obtain an increasing sequence $x_{1} \leq x_{2} \leq \cdots \leq x_{k_{N}}$ of real parts, and we plotted the points $\left(x_{j}, j\right)$. Thus, steep parts of the density graph indicate that

\footnotetext{
${ }^{9}$ In general, times $\varepsilon^{1-\omega_{u}} P\left(\log \varepsilon^{-1}\right)$, where $P$ is a polynomial of degree one less than the multiplicity of $\omega_{u}$ as a pole of $\zeta_{\mathcal{L}}$. Recall that $D$ is always a simple pole.
} 

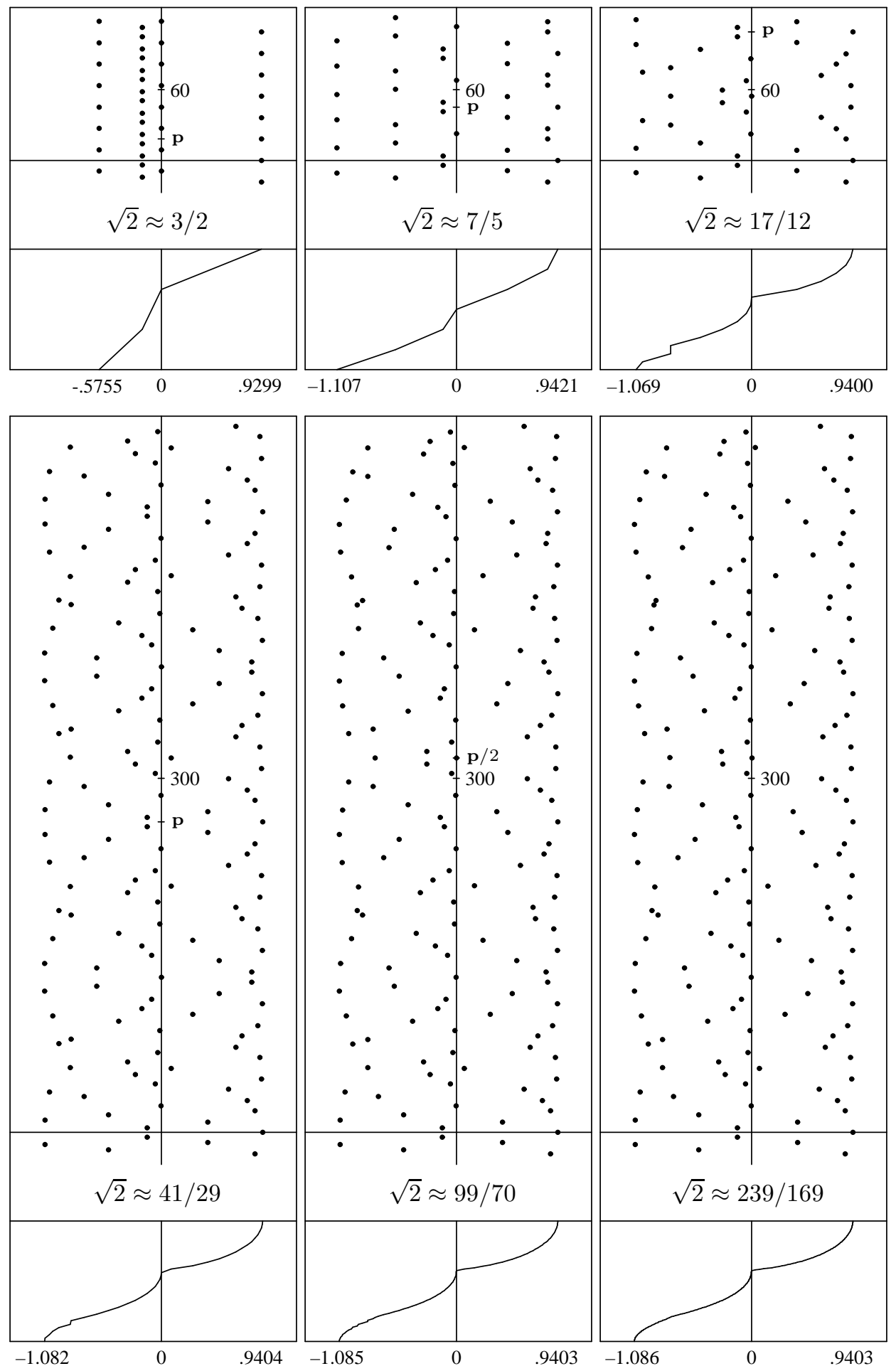

Figure 2. Six stages of approximation of the complex dimensions of the nongeneric nonlattice string of Example 4.5, with $r_{1}=2^{-1}$, $r_{2}=2^{-2}$ and $r_{3}=2^{-1-\sqrt{2}}$. Lower parts: The density graph of the real parts of the complex dimensions. 
there are many complex dimensions with almost the same real part, whereas flat parts indicate that the sequence $x_{j}, x_{j+1}, \ldots$ increases relatively fast.

4.2. The Nonlattice Case. The nonlattice case is when the group $G$ defined at the beginning of $\S 4.1$ is dense in $\mathbb{R}_{+}^{*}$. Moreover, the fractal string $\mathcal{L}$ is said to be a generic nonlattice string if the cardinality $M=\#\left\{r_{1}, \ldots, r_{N}\right\}$ is at least two and equals the rank of $G$ as a free abelian group. In this case, the lengths are maximally dispersed in $\mathbb{R}_{+}^{*}$. Typically, their multiplicity still grows exponentially, but at a slower rate than in the nongeneric nonlattice case or the lattice case.

The geometry of a nonlattice self-similar string is much more elusive. Indeed, the only way to obtain detailed information about the volume of the tubular neighborhoods might be via the geometric zeta function, the complex dimensions, and the explicit formula (2.4). Much like a real number can be approximated by a suitable sequence of rational numbers, obtained by means of its continued fraction expansion (see [Lap-vF4] and [HaWr,Sch]), the set of lattice strings is dense in the space of all self-similar strings. By an explicit Diophantine approximation procedure (see [Lap-vF2, Thm. 2.26, Lap-vF4]), we can approximate the nonlattice string $\mathcal{L}$ by a sequence of lattice strings $\left\{\mathcal{L}^{(n)}\right\}_{n=1}^{\infty}$ with oscillatory period $\mathbf{p}_{n}=2 \pi q_{n} / \log r^{-1}$, for larger and larger integers $q_{n}$. Here, $r=r_{1}$ is the first (i.e., largest) self-similarity ratio of $\mathcal{L}$. Hence we can approximate, in the sense of [Lap-vF2, Def. 2.5], the set of complex dimensions $\mathcal{D}_{\mathcal{L}}$ of $\mathcal{L}$ by the corresponding sets of complex dimensions $\mathcal{D}_{n}=\mathcal{D}_{\mathcal{L}^{(n)}}$. In particular, the complex dimensions of a nonlattice string exhibit a quasiperiodic pattern, as explained in the following counterpart of Theorem 4.1 (see [Lap-vF2, Thms. 2.13,6.20], along with [Lap-vF4]):

Theorem 4.2 (Nonlattice Case: Quasiperiodic Patterns). Let $\mathcal{L}$ be a nonlattice self-similar string. Then $\mathcal{L}$ has infinitely many complex dimensions (with positive real part). The density of the complex dimensions (counted with multiplicity) is

$$
\#\left\{\omega \in \mathcal{D}_{\mathcal{L}}: 0 \leq \operatorname{Im} \omega \leq T\right\} \leq \frac{\log r_{N}^{-1}}{2 \pi} T+O(1), \quad \text { as } T \rightarrow \infty,
$$

with equality if $\mathcal{L}$ has only one gap. Further, $D$ is the only complex dimension with real part equal to $D$, and the other complex dimensions lie in a horizontally bounded strip $\sigma_{l} \leq \operatorname{Re} \omega<D$, where $\sigma_{l}=\inf \left\{\operatorname{Re} \omega: \sum_{j=1}^{N} r_{j}^{\omega}=1\right\} .{ }^{10}$

Each complex dimension $\omega_{u}$ of $\mathcal{L}$ gives rise to a sequence of complex dimensions $\omega$ of $\mathcal{L}$, close to the sequence (4.1). Let $r_{1}$ denote the largest scaling ratio of $\mathcal{L}$. Then the period of this sequence is $\mathbf{p}=2 \pi / \log r_{1}^{-1}$. Further, for certain multiples of this oscillatory period, $\mathbf{p}_{n}=q_{n} \mathbf{p}=2 \pi q_{n} / \log r_{1}^{-1}$, where the sequence of integers $q_{n}$ depends on the arithmetic nature of the scaling ratios and is such that $q_{n} \rightarrow \infty$ as $n \rightarrow \infty$, one obtains subsequences that lie arbitrarily close to the sequence (4.1), with an oscillatory period of $q_{n} \mathbf{p}$ instead of $\mathbf{p}$.

We see that nonlattice complex dimensions 'lie on infinitely many vertical lines' with larger and larger oscillatory period. Thus we have an infinite spectrum of selfsimilarity in the nonlattice case.

\footnotetext{
${ }^{10}$ For a generic nonlattice string it can be shown that $\sigma=\sigma_{l}$ is the unique real solution to the equation $1+r_{1}^{\sigma}+\cdots+r_{N-m-1}^{\sigma}=m r_{N}^{\sigma}$, where $m=\#\left\{j: r_{j}=r_{N}\right\}$ is the multiplicity of $r_{N}$. Moreover, in most cases, including the generic nonlattice case, the infimum is not attained. In fact, it is possible that the infimum is only attained in the lattice case.
} 
The following theorem refines the information about the complex dimensions close to the sequence $\{D+k i \mathbf{p}: k \in \mathbb{Z}\}$ when $N=2$. There are two scaling ratios $r_{1}$ and $r_{2}=r_{1}^{\alpha}$, for some irrational number $\alpha>1$, and we write

$$
f(s)=1-r_{1}^{s}-r_{2}^{s}=1-r_{1}^{s}-r_{1}^{\alpha s},
$$

so that $f^{\prime}(D)=\left(r_{1}^{D}+\alpha r_{2}^{D}\right) \log r_{1}^{-1}$. See [Lap-vF4, Thm. 4.3] for details and a proof.

Theorem 4.3 (Dimensions above $D)$. For $k \in \mathbb{Z}$, let $x=2 \pi i(k \alpha-l)$, where $l$ is the integer nearest to $k \alpha$. Then the complex dimension of $\mathcal{L}$ close to $D+i k \mathbf{p}$, $\mathbf{p}=2 \pi / \log r_{1}^{-1}$, is approximated by

$$
\omega=D+k i \mathbf{p}-\frac{r_{2}^{D}}{f^{\prime}(D)} x+\frac{\left(\log r_{1}\right)^{2} r_{1}^{D} r_{2}^{D}}{2 f^{\prime}(D)^{3}} x^{2}+O\left(x^{3}\right) .
$$

Note that the first correction term (of order $x$ ) is purely imaginary, and the second correction is negative of order $x^{2}$ (since $\left.x^{2}<0\right)$. Hence we see again that Re $\omega<D$. We refer to [Lap-vF4, Eq. (4.16)] for the general case when $N>2$.

This theorem allows us to obtain a dimension-free region, recalled in Equation (5.2) below. Using the explicit formula (2.4) and this dimension free region, we obtain, by techniques explained in [Lap-vF3], that for every (small) $\delta>0$,

$$
V(\varepsilon)=\mathcal{M} \varepsilon^{1-D}\left(1+O\left(\frac{1}{|\log \varepsilon|^{(N-1) / 2-\delta}}\right)\right), \quad \text { as } \varepsilon \rightarrow 0^{+} .
$$

The error estimate in (4.8) is best possible when the system $\log r_{j} / \log r_{1}(j=$ $2, \ldots, N)$ is badly approximable in the sense of $\S 5$.

It follows from (4.8) and a computation of the residue in (2.5) that a nonlattice self-similar string is Minkowski measurable, with a Minkowski content

$$
\mathcal{M}=\mathcal{M}(D ; \mathcal{L})=\frac{2^{1-D} L^{D}\left(g_{1}^{D}+\cdots+g_{K}^{D}\right)}{D(1-D)\left(r_{1}^{D} \log r_{1}^{-1}+\cdots+r_{N}^{D} \log r_{N}^{-1}\right)} .
$$

REMark 4.4. (a) The statement of Theorem 4.2 regarding the Minkowski measurability of nonlattice self-similar strings (i.e., of nonlattice self-similar sets in $\mathbb{R}$ ) was first obtained independently in [Lap3] and by Falconer in [Fa3], by means of the Renewal Theorem [Fel, Ch. XI]. It has recently been extended to any (suitable) nonlattice self-similar set in $\mathbb{R}^{d}(d>1)$ by Gatzouras in [Gat], thereby proving the nonlattice case of the geometric part of [Lap3, Conj. 3, p. 163] concerning selfsimilar drums. (See also [Lap-vF2, Conj. 10.13, p. 209] for an extension of part of that conjecture to the more general 'tube formulas' obtained in the context of the theory of complex dimensions.)

(b) Part of the results of [Lap-vF2] concerning the complex dimensions of selfsimilar strings was extended to random self-similar strings (and other random fractal strings, such as the zero set of Brownian motion) by Ben Hambly and the first author in [HamLap]. A rich variety of behaviors of the complex dimensions is encountered in this context.

4.3. Nonlattice Strings: Examples and Open Problems. In order to illustrate Theorem 4.2 and to formulate some problems and a conjecture regarding the nonlattice case (generic and nongeneric), we discuss three examples of nonlattice strings. Our first example is a nongeneric nonlattice string. 
EXAmPle 4.5 (A Nongeneric Nonlattice String). Consider the self-similar string with scaling ratios $r_{1}=1 / 2, r_{2}=1 / 4, r_{3}=2^{-1-\sqrt{2}}$ and one gap $g=1 / 4-r_{3}$. Figure 2 gives six approximations to its complex dimensions (along with an associated graph of the density of their real parts in the lower diagrams, to be explained below) corresponding to the successive approximations to $\sqrt{2}$ (obtained by the continued fraction expansion $[\mathrm{HaWr}]): \frac{p_{n}}{q_{n}}=\frac{3}{2}, \frac{7}{5}, \frac{17}{12}, \frac{41}{29}, \frac{99}{70}, \frac{239}{169}, \cdots \rightarrow \sqrt{2}$. For example, the first approximation gives the lattice string with scaling ratios $\tilde{r}_{1}=2^{-1}$, $\tilde{r}_{2}=2^{-2}, \tilde{r}_{3}=2^{-5 / 2}$, the complex dimensions of which are the solutions to the equation $z^{2}+z^{4}+z^{5}=1,2^{-\omega / 2}=z$. The oscillatory period of this lattice approximation is $\mathbf{p}=4 \pi / \log 2$. One sees the development of a quasiperiodic pattern: the complex dimensions of the nonlattice string are well approximated by those of a lattice string for a certain finite number of periods of the lattice approximation. Then that periodic pattern gradually disappears, and a new periodic pattern, approximated by the next lattice approximation, emerges.

It takes fairly large approximations to see this quasiperiodic pattern. For example, the complex dimensions of the three nonlattice strings of Examples 4.5 and 4.7 are respectively approximated to within a distance $\frac{1}{10}$ by the points in the three diagrams of Figure 3 for only about two periods $\mathbf{p}$ of the respective lattice strings (four periods for the last one, since $\mathbf{p}$ is half the size, as explained in Example 4.7). This is found by adapting the proof of [Lap-vF4, Thm. 3.6] to these strings, since a direct application of this theorem would give only half a period of good approximation. The number of periods (of the lattice string) for which the approximation is good does grow linearly in the denominator of the approximation. Note that the period itself also grows like this denominator.

The left diagram in Figure 3 gives an impression on a larger scale of the quasiperiodic behavior of the complex dimensions of the nongeneric nonlattice string of Example 4.5. The other two diagrams in this figure give the complex dimensions of the two generic nonlattice strings of Example 4.7 below. One sees that the complex dimensions in the left diagram are much denser to the left of vanishing real part. One sees this even more clearly in Figure 4, where the cumulative density of the real parts is graphed for these three self-similar strings. We have no explanation for this apparent 'phase transition'. We formulate this question as a problem (see Theorem 4.2 and footnote 10 for the definition of $\sigma_{l}$ ):

Problem 4.6 (Transition in the Nongeneric Nonlattice Case). A nongeneric nonlattice string has a vertical line of transition inside the strip $\sigma_{l} \leq \operatorname{Re} s \leq D$, to the left of which the density of the real parts is infinitely higher than to the right. Such a transition does not occur for generic nonlattice strings.

Thus, for the nongeneric nonlattice string of Example 4.5, this transition occurs at $\operatorname{Re} s=0$, as indicated by the corner of the density graph at this point, and the vertical part of the graph to the left of $\operatorname{Re} s=0$. Moreover, from other numerical evidence, it seems that this line of transition often occurs at $\operatorname{Re} s=0$.

Example 4.7 (Two Generic Nonlattice Strings). We also include in Figures 3 and 4 the analogous diagrams for two generic nonlattice strings. These are respectively the nonlattice strings with two scaling ratios $r_{1}=1 / 2$ and $r_{2}=2^{-1-\sqrt{2}}$ (the middle diagrams in both figures) and $r_{1}=1 / 4$ and $r_{2}=2^{-1-\sqrt{2}}$ (the right diagrams in both figures). Each of these strings has a single gap of length respectively 


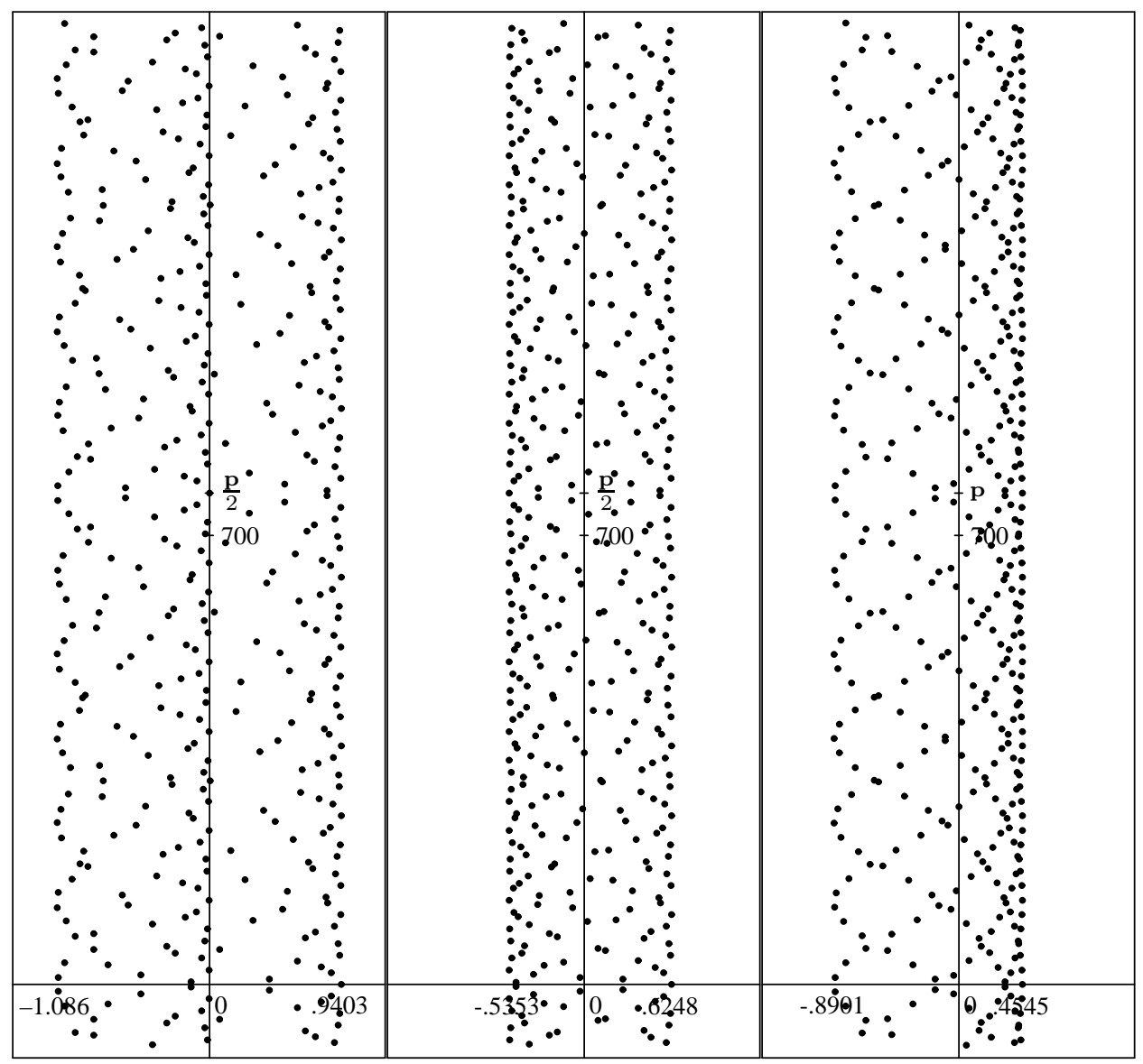

Figure 3. An impression of the quasiperiodic pattern of complex dimensions on a larger scale, for the self-similar string of Example 4.5 (left) and those of Example 4.7 (center and right).

$g_{1}=1 / 2-2^{-1-\sqrt{2}}$ and $g_{1}=3 / 4-2^{-1-\sqrt{2}}$. Note that for the second string, with $r_{1}=1 / 4$ and $r_{2}=2^{-1-\sqrt{2}}$, the approximation $1+\sqrt{2} \approx \frac{408}{169}$ leads to the equation

$$
z^{2 \cdot 169}+z^{408}=1, \quad 2^{-\omega / 169}=z .
$$

Since this is an equation in $z^{2}$, the oscillatory period of the lattice equation, namely, $169 \cdot \pi / \log 2$, is only slightly larger than that of the lattice string corresponding to the previous approximation, $1+\sqrt{2} \approx \frac{169}{70}$, which is $70 \cdot 2 \pi / \log 2$.

We see in the Figures 3 and 4 that the complex dimensions of the nongeneric and generic nonlattice strings of Examples 4.5 and 4.7 are much denser near the boundaries $\operatorname{Re} s=\sigma_{l}$ and $\operatorname{Re} s=D$ of the critical strip. Indeed, in [Lap-vF4], we have shown that given any nonlattice string with two scaling ratios, there exists an explicit positive constant $C_{5}$ (expressed in terms of $r_{1}$ and $r_{2}$ ), such that the density graph of the real parts is approximated by the graph $y=1-C_{5} \sqrt{D-x}$, for $x \leq D$ in a small neighborhood to the left of $D$. More generally, if the rank of $G$ is $v$ (so $v \leq M$, and $v=M$ in the generic nonlattice case, see $\S 4.1$ ), then the density 


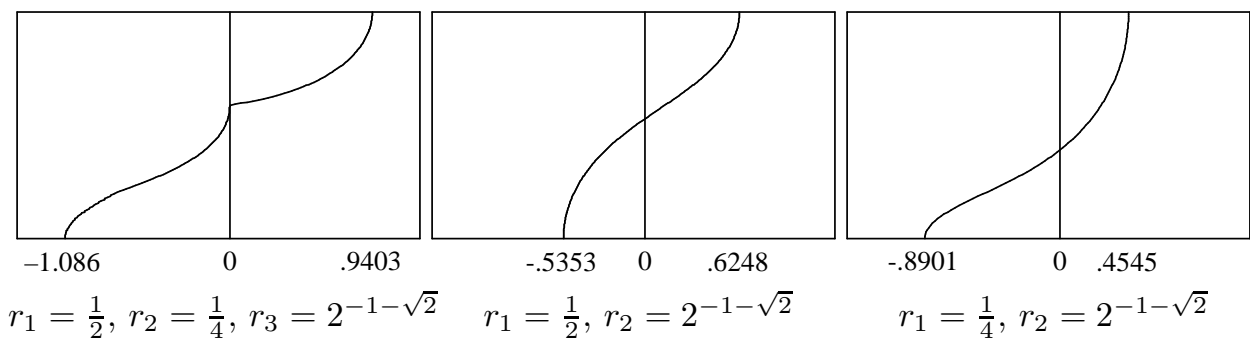

Figure 4. Comparison of the densities of the real parts for the nongeneric nonlattice string of Example 4.5 (left) and the two generic nonlattice strings of Example 4.7 (center and right).

graph is approximated by the graph $y=1-C_{6}(D-x)^{(v-1) / 2}$. Hence, if there are three multiplicatively independent scaling ratios $(v=M=3)$, then the density at the boundary is comparable to the average density, whereas for $v \geq 4$, the complex dimensions thin out near the boundary of the 'critical strip' $\sigma_{l} \leq \operatorname{Re} \omega \leq D$. Indeed, numerical evidence suggests the following problem, further evidence for which will be provided in $\S 5$, in connection with the properties of Diophantine approximation of the ratios $\log r_{j} / \log r_{1}$. We refer to [Lap-vF4, Rem. 4.14] for more details. Also see $\S 5$ for some information about bad approximability.

Problem 4.8 (Complex Dimensions Close to One Line). The complex dimensions of a nonlattice string with a large number of different scaling ratios are concentrated around a single line within its critical strip. That is, the graph of the density of the real parts of the complex dimensions has a narrow steep part, and two flat parts to the left and the right. Moreover, if this phenomenon occurs, it may be most apparent when the scaling factors $r_{j}$ are such that the ratios $\log r_{j} / \log r_{1}$ $(j=2, \ldots, N)$ are badly approximable.

We have shown in [Lap-vF4, Thm. 8.1] that for any nonlattice string, the real parts of the complex dimensions form a set without isolated points. In [Lap$\mathrm{vF} 2, \mathrm{Ch} .10]$, we proposed as a definition of fractality the existence of at least one nonreal complex dimension with positive real part. Since nonreal complex dimensions give oscillatory terms in the explicit formula for the tubular neighborhoods, that is, terms that are 'self-similar' under a (multiplicative) scaling, this theorem can be phrased as saying that a nonlattice string is fractal in a perfect set of dimensions. The following conjecture even says that a nonlattice string is fractal in every dimension in $\left[\sigma_{l}, D\right]$ (see Theorem 4.2 for the definition of $\sigma_{l}$ ).

Conjecture 4.9 (Density of the Real Parts). Let $\mathcal{L}$ be a nonlattice string. Then the real parts of its complex dimensions form a set that is dense in the connected interval $\left[\sigma_{l}, D\right]$.

We sketch the idea for a possible proof of this conjecture when $N=2$. By Equation (4.7), one can find a complex dimension with real part arbitrarily close to $D$. Moreover, for every complex dimension $\omega$ there exists an analogue of Equation (4.7), yielding a sequence of complex dimensions close to the line $\omega+k i \mathbf{p}$ $(k \in \mathbb{Z})$. The main correction term, of order $x$, will in general not be purely imaginary, as it is for $\omega=D$, and for suitable values of $k$, the real part of $x$ will be 
negative. Continuing in this manner, given any $\varepsilon>0$, one can find a sequence of complex dimensions, starting at $D$, the real parts of which form a decreasing sequence of numbers that differ by less than $\varepsilon$. This would prove the density of real parts in an interval. It remains to establish a suitable version of Equation (4.7) (and its analogues for $N>2$ ), for every complex dimension, and to analyze it. Of course, the argument that the real part of $x$ is negative for suitable values of $k \in \mathbb{Z}$ breaks down when one reaches the left boundary of the 'critical strip'. There, $x$ will become purely imaginary (like at $D$ ), and the second correction term will become positive. Note, however, that there is no complex dimension with real part equal to $\sigma_{l}$.

\section{Dimension-Free Regions}

DEFINITION 5.1. An open domain in the complex plane is a dimension-free region for the string $\mathcal{L}$ if it contains the line $\operatorname{Re} s=D$ and the only pole of $\zeta_{\mathcal{L}}$ in that domain is $s=D$.

In the situation of Theorem 4.3, when $N=2$, the dimension-free region depends on the approximability by rationals of $\alpha$, where $\alpha=\log r_{2} / \log r_{1}$ as in Equation (4.6). Assume that $\alpha$ is badly approximable: $|q \alpha-p| \geq C_{7} / q$ for all integers $p$ and $q$ (see, e.g., [DoKr] $).{ }^{11}$ Put $C_{8}=\pi^{4}\left(r_{1} r_{2}\right)^{D} /\left(2 f^{\prime}(D)^{3}\right)$, where $f$ is the function defined in (4.6). Then $\left\{\sigma+i t \in \mathbb{C}: \sigma>D-C_{8} C_{7}^{2} t^{-2}\right\}$ is a dimension-free region for $\mathcal{L}$. More generally, let $g: \mathbb{R}_{+} \rightarrow(0,1]$ be a nonincreasing function that measures the approximability of $\alpha$ by rationals: $|q \alpha-p| \geq g(q) / q$ for all integers $p$ and $q$. Write $w_{1}=\log r_{1}^{-1}$. Then $\mathcal{L}$ has a dimension-free region of the form

$$
\left\{\sigma+i t \in \mathbb{C}: \sigma>D-C_{8} g^{2}\left(w_{1} t / 2 \pi\right) t^{-2}\right\} .
$$

In the general case, when $N \geq 2$, the dimension-free region is best (widest) if the ratios $\log r_{j} / \log r_{1}(j=2, \ldots, N)$ are badly approximable. In that case, $\mathcal{L}$ has a dimension-free region of the form (see [Lap-vF3-4])

$$
\left\{\sigma+i t \in \mathbb{C}: \sigma>D-C_{9} t^{-2 /(N-1)}\right\},
$$

where the constant $C_{9}$ depends only on $r_{1}, \ldots, r_{N}$. On the other hand, if those ratios are better approximable, then the dimension-free region is worse, in the sense that there are complex dimensions that lie very close to the vertical line $\operatorname{Re} s=D$.

From these results it is also clear that in general, depending on the properties of simultaneous Diophantine approximation of the scaling ratios, nonlattice strings with a larger number of scaling ratios have a wider (and hence better) dimension-free region. This reinforces the belief in Problem 4.8. For more information regarding the results of $\S 5$ and their proofs, as well as for related results also based in part on Diophantine approximation, we refer to [Lap-vF3 \& $4, \S 7]$.

\section{Self-Similar Dynamical Systems}

We refer the interested reader to [Lal2, Lap-vF3-4,ParP1-2], and the relevant references therein, for more information on the dynamical systems considered here.

\footnotetext{
${ }^{11}$ Equivalently, $\alpha$ has bounded partial quotients in its continued fraction expansion.
} 
Regarding earlier results on the Prime Orbit Theorem in a related context, we mention, in particular, the early works of Huber [Hub], Sinai [Si], and Margulis [Mar] (see the historical note in [BedKS, p. 154]) and the more recent works by Parry and Pollicott [ParP1\&2, Ch. 6] as well as by Lalley [Lal2].

For simplicity and because this is the situation that is best understood so far, we consider here the dynamical counterpart of self-similar strings with a single gap $(K=1)$. It would be interesting to extend our formalism to include the multiple gap case. This can possibly be done within the setting of [HamLap] where 'GaltonWatson' random trees are used to study the zeta functions of random strings.

Let $N \geq 0$ be an integer and let $\Omega=\{1, \ldots, N\}^{\mathbb{N}}$ be the space of sequences over the alphabet $\{1, \ldots, N\}$. Let $\mathfrak{w}: \Omega \rightarrow(0, \infty]$ be a function, called the weight. On $\Omega$, we have the left shift $\tau$, given on a sequence $z=\left(z_{n}\right)$ by $(\tau z)_{n}=z_{n+1}$. We define the suspended flow $\mathcal{F}_{\mathfrak{w}}$ on the space $[0, \infty) \times \Omega$ as the following continuous-time dynamical system (see [Lap-vF3, Fig. 1, p. 117]):

$$
\mathcal{F}_{\mathfrak{w}}(t, z)= \begin{cases}(t, z) & \text { if } 0 \leq t<\mathfrak{w}(z) \\ \mathcal{F}_{\mathfrak{w}}(t-\mathfrak{w}(z), \tau z) & \text { if } t \geq \mathfrak{w}(z)\end{cases}
$$

Given a finite sequence $z_{1}, z_{2}, \ldots, z_{l}$, we let $z=z_{1}, z_{2}, \ldots, z_{l}, z_{1}, z_{2}, \ldots, z_{l}, \ldots$ be the corresponding periodic sequence, and $\mathfrak{p}=\left\{z, \tau z, \tau^{2} z, \ldots\right\}$ the associated finite orbit of $\tau$, of length $\# \mathfrak{p}$. Thus the length of $\mathfrak{p}$ is a divisor of $l$. The total weight of $\mathfrak{p}$ is $\mathfrak{w}_{\text {tot }}(\mathfrak{p})=\sum_{z \in \mathfrak{p}} \mathfrak{w}(z)$. We define the dynamical zeta function (or Bowen-Ruelle zeta function [Bo, R, ParP2]) of $\mathcal{F}_{\mathfrak{w}}$ by the Euler-type product

$$
\zeta_{\mathfrak{w}}(s)=\prod_{\mathfrak{p}} \frac{1}{1-e^{-\mathfrak{w}_{\text {tot }}(\mathfrak{p}) s}},
$$

where $\mathfrak{p}$ runs over all finite orbits of $\tau$.

The function $\psi_{\mathfrak{w}}(x)=\sum_{k \mathfrak{w}_{\text {tot }}(\mathfrak{p}) \leq \log x} \mathfrak{w}_{\text {tot }}(\mathfrak{p})$ is the analogue of the function $\psi(x)=\sum_{p^{k} \leq x} \log p$ in number theory, which counts the prime powers with a weight $\log p .^{12}$ It counts the periodic orbits of $\mathcal{F}_{\mathfrak{w}}$ and their multiples by their total weight. It may be referred to as the weighted prime orbit counting function and is related to the dynamical zeta function by $-\zeta_{\mathfrak{w}}^{\prime}(s) / \zeta_{\mathfrak{w}}(s)=\int_{0}^{\infty} x^{-s} d \psi_{\mathfrak{w}}(x)$, for $\operatorname{Re} s>D$. The poles of the logarithmic derivative $\zeta_{\mathfrak{w}}^{\prime} / \zeta_{\mathfrak{w}}$ are called the dynamical complex dimensions of $\mathcal{F}_{\mathfrak{w}}$. Thus the dynamical complex dimensions are the poles and the zeros of $\zeta_{\mathfrak{w}}$, counted without multiplicity. (See [Lap-vF3] for more information.)

The flow $\mathcal{F}_{\mathfrak{w}}$ is said to be self-similar if $N \geq 2$ and the weight function $\mathfrak{w}$ depends only on the first letter of the sequence at which it is evaluated. We then define the scaling ratios of $\mathcal{F}_{\mathfrak{w}}$ by $r_{j}=\exp (-\mathfrak{w}(j, j, \ldots))$ for $j=1, \ldots, N$. Much as in $\S 4$, the flow is said to be lattice if the group $G$ defined in $\S 4.1$ has rank 1 , and nonlattice otherwise. We associate with $\mathcal{F}_{\mathfrak{w}}$ a self-similar fractal string with these scaling ratios and one gap $g=1-r_{1}-\cdots-r_{N}$ and total length such that $g L=1$ (we need to require that $r_{1}+\cdots+r_{N}<1$ ). Then we have $\zeta_{\mathfrak{w}}(s)=\zeta_{\mathcal{L}}(s)$ (see $\S 3$ and [Lap-vF3, Thm. 2.10]). Since $\zeta_{\mathfrak{w}}$ has no zeros in this case, the dynamical complex dimensions of a self-similar flow coincide with the geometric complex dimensions of the corresponding self-similar string, but they are counted without multiplicity.

\footnotetext{
${ }^{12}$ In the literature, $\psi$ is traditionally referred to as the 'von Mangoldt function' and is often used, in particular, to prove the classical Prime Number Theorem; see, e.g., [In, Pat, Ti].
} 
If $D$ is the only complex dimension on the line $\operatorname{Re} s=D$, then we deduce (from the explicit formula for the prime orbit counting function) a Prime Orbit Theorem for $\mathcal{F}_{\mathfrak{w}}: \psi_{\mathfrak{w}}(x)=x^{D} / D+o\left(x^{D}\right)$, as $x \rightarrow \infty$. This is the analogue of the famous Prime Number Theorem, which can be stated as $\psi(x)=x+o(x)$. In the nonlattice case, using the dimension-free region (5.2) and the techniques explained in [LapvF3], we even obtain for every (small) $\delta>0$ the following Prime Orbit Theorem with Error Term:

$$
\psi_{\mathfrak{w}}(x)=\frac{x^{D}}{D}\left(1+O\left(\frac{1}{(\log x)^{(N-1) / 2-\delta}}\right)\right), \quad \text { as } x \rightarrow \infty .
$$

This estimate is best possible if the system $\left\{\log r_{j} / \log r_{1}: j=2, \ldots, N\right\}$ is badly approximable (as explained for $N=2$ in $\S 5$ ). We note that in [Lap-vF3], we also obtain more precise explicit formulas for the counting function $\psi_{\mathfrak{w}}$.

\section{The Complex Dimensions as the Spectrum of Shifts}

In the simplest case of the Cantor string, discussed in the introduction and in Example 3.2, it is easy to see that a shifted copy, CS $+x$, overlaps the Cantor string only for shifts over $2 \cdot 3^{-n}, n=0,1, \ldots$, or suitable combinations of such shifts: $x=\sum_{n=0}^{\infty} a_{n} 3^{-n}, a_{n}= \pm 2$. For all other values of the real number $x$, there is no overlap: $\mathrm{CS} \cap(\mathrm{CS}+x)=\emptyset$. Moreover, the Minkowski dimension (or Hausdorff dimension) of the intersection is always $D=\log _{3} 2$ and its Minkowski content (or Hausdorff measure) is $2^{k}$ times smaller than that of the Cantor set itself, where $k$ is the number of nonzero digits of $x$ in the above representation. This information suffices to reconstruct the Cantor set and its complex dimensions.

In general, let $F$ be a self-similar fractal subset of the real line (see Remark 3.1). Let $I_{\varepsilon}$ denote the interval $(-\varepsilon, \varepsilon)$. To measure the overlap of $F$ with a shifted copy of it, we consider the function ${ }^{13} f(\varepsilon, x)=\left|\left(F+I_{\varepsilon}\right) \cap\left(F+I_{\varepsilon}+x\right)\right|$, for $x \in \mathbb{R}$. We then construct the 'dimension-like function'

$$
D(x)=\inf \left\{d \geq 0: f(\varepsilon, x)=O\left(\varepsilon^{1-d}\right) \text { as } \varepsilon \rightarrow 0^{+}\right\}
$$

and the 'upper Minkowski content-like function'

$$
\mathcal{M}^{*}(x)=\limsup _{\varepsilon \rightarrow 0^{+}} f(\varepsilon, x) \varepsilon^{D(x)-1} .
$$

We expect that these functions exhibit the following behavior. In the lattice case, $D(x)>0$ for a bounded discrete set of values. The function $\mathcal{M}^{*}(x)$ is discontinuous at each of these values, and continuous and vanishing on the complement. In the nonlattice case, $D(x)>0$ for every $x$ in a countable dense subset of a compact connected interval and $\mathcal{M}^{*}(x)$ is continuous. The complex dimensions of $F$ can be recovered from the functions $D(x)$ and $\mathcal{M}^{*}(x)$. We hope to develop these ideas in subsequent work.

\section{A Cohomological Interpretation of the Complex Dimensions}

We have suggested in [Lap-vF2, $\S 10.5]$ that there should exist a notion of 'complex cohomology' that could be associated to both arithmetic and self-similar geometries and have proposed a possible dictionary towards it, building in part upon

\footnotetext{
${ }^{13}$ See footnote 6 for the notation $F+I_{\varepsilon}$.
} 
the analogy between lattice strings (or more generally, lattice self-similar geometries) and curves (or varieties) over finite fields [Wei1-3, ParsSh, I]. We have proposed, in particular, to construct a 'cohomology theory', which to each (dynamical) complex dimension $\omega$ of such a geometry, would associate a nontrivial cohomology group $H^{\omega}$ (with coefficients in the field of complex numbers). In general, $H^{\omega}$ would be expected to be an infinite dimensional Hilbert space. This should be the case, for example, for the cohomology spaces associated with a nonlattice string (or more generally, with a nonlattice self-similar geometry). A possible way of developing such a theory was proposed at the very end of [Lap-vF2, 110.5$]$. It relies on a suitable equivalence relation between the Dirichlet series (or 'zeta functions') attached to these geometries; see [Lap-vF2, p. 220].

8.1. Fractal Geometries and Finite Geometries. The geometric zeta function of a fractal string can have complex dimensions of higher multiplicity, like in Example 3.4 above. Hence $\zeta_{\mathcal{L}}(s)$ should be compared to the zeta function of a variety over a finite field, and not for example to its logarithmic derivative. This is confirmed by the fact that the residue at a complex dimension is quite arbitrary, and not an integer in general. Another confirmation is that the logarithmic derivative of the geometric zeta function is the logarithmic derivative of the dynamical zeta function (as shown in [Lap-vF3] and recalled in $\S 6$ above), which is the generating function of the counting function of the periodic orbits of a dynamical system. This corresponds to the logarithmic derivative of the zeta function of a variety, which is the generating function of the counting function of the periodic orbits of the Frobenius automorphism, as explained in $\S 1.1$. On the other hand, for the simplest self-similar strings (i.e., those without gaps [Lap-vF2]), the geometric zeta function has only poles and no zeros. This would mean that there is only 'even dimensional cohomology' in that case, but the correct interpretation is as yet unclear.

We complete the dictionary proposed in [Lap-vF2, $\$ 10.5]$ with the following three tables. The first table summarizes the analogies between the corresponding zeta functions.

\begin{tabular}{ll} 
self-similar geometries & 'finite' geometries \\
\hline lattice string & variety $V$ over the field $\mathbb{F}_{q}$ \\
nonlattice string $\mathcal{L}$ & infinite dimensional variety \\
Cantor string & affine rational variety \\
geometric zeta function $\zeta_{\mathcal{L}}$ & zeta function $\zeta_{V}$ \\
$\quad$ counts lengths & counts divisors \\
lattice case is periodic & the zeta function is periodic \\
period p is typically large & period is typically small \\
nonlattice: $\mathbf{p} \rightarrow \infty$ & period $\rightarrow 0$ if $\# \mathbb{F}_{q} \rightarrow \infty$ \\
$\quad($ i.e., 'characteristic' $\rightarrow 1)$ & \\
residue at $D($ Minkowski & residue at $n($ dimension) \\
dimension) gives $\mathcal{M}(D ; \mathcal{L})$ & gives the class number
\end{tabular}

TABLE 1. Self-similar fractal geometries vs. varieties over finite fields: Analogies between the zeta functions.

We explain the sense in which $\mathbf{p} \rightarrow \infty$ for nonlattice strings, and the connection with the characteristic. Recall that the oscillatory period of a lattice string 
with scaling ratios $r_{j}=r^{k_{j}}, j=1, \ldots, N$ (for positive integers $k_{1}, \ldots, k_{N}$ without common factor and $r \in(0,1))$ is $\mathbf{p}=\frac{2 \pi}{\log r^{-1}}$. It is the period of its geometric zeta function: $\zeta_{\mathcal{L}}(s)=\zeta_{\mathcal{L}}(s+i \mathbf{p})$, for $s \in \mathbb{C}$. On the other hand, if $\mathcal{L}$ is a nonlattice string, and $r_{j} \approx r^{k_{j}}$ gives a lattice approximation, then $\zeta_{\mathcal{L}}(s)$ and $\zeta_{\mathcal{L}}(s+i n \mathbf{p})$ are close, for $n$ not too large. The next (better) lattice approximation to $\mathcal{L}$ has larger values for the integers $k_{j}(j=1, \ldots, N)$ and a value of $r$ that is closer to 1 . Hence, its oscillatory period is larger (see $\S 4$ for more details). In that sense, $\mathbf{p} \rightarrow \infty$ for nonlattice strings, as $\mathbf{p}$ runs through the values of the oscillatory periods of lattice approximations. For a variety (of dimension $n$ ) over the finite field $\mathbb{F}_{q}$, on the other hand, the zeta function is periodic with period $\mathbf{p}=\frac{2 \pi}{\log q}$. Again, the zeta function of $V$ is periodic: $\zeta_{V}(s)=\zeta_{V}(s+i \mathbf{p})$, for $s \in \mathbb{C}$. Here, $q$ is a power of a prime number, the characteristic of the finite field. Hence the period is small typically. However, the limit as $q \downarrow 1$ (i.e., if the characteristic of $\mathbb{F}_{q}$ tended to 1 , if this were possible), corresponds to the limit $r \uparrow 1$ that we see for nonlattice strings.

The next table summarizes the expected properties of a cohomology theory for self-similar fractal strings (and sets).

\begin{tabular}{ll} 
self-similar geometries & 'finite' geometries \\
\hline poles from the scaling ratios & poles from even cohomology \\
zeros from the gaps & zeros from odd cohomology \\
lattice case is periodic & zeta function is periodic \\
nonlattice cohomology is & the cohomology collapses to \\
$\quad$ infinite dimensional & a finite-dimensional one
\end{tabular}

TABLE 2. Self-similar fractal geometries vs. varieties over finite fields:

Cohomological aspects.

Finally, Table 3 presents the dynamical analogies between self-similar flows and the Frobenius flow of a variety.

\begin{tabular}{cc} 
self-similar geometries & 'finite' geometries \\
\hline dynamical flow & Frobenius flow \\
dynamical zeta function $-\zeta_{\mathcal{L}}^{\prime} / \zeta_{\mathcal{L}}$ & $-\zeta_{V}^{\prime} / \zeta_{V}$ counts the Frobenius \\
counts the closed orbits & or Galois orbits of points \\
Euler-type product connects & Euler product connects \\
orbits with lengths & orbits with divisors \\
$r^{\omega}$ (poles) are solutions & $q^{\omega}$ (zeros and poles) are \\
to $(3.2)$ & eigenvalues of Frobenius \\
number of lines is $k_{N}=$ degree & number of lines is $2 n+1$, \\
of $(3.2)$ & $n=\operatorname{dim} V$
\end{tabular}

TABLE 3. Self-similar fractal geometries vs. varieties over finite fields:

Dynamical aspects.

REMARK 8.1. One aspect of Table 2 that we have not developed in this paper is that the dichotomy lattice vs. nonlattice also exists for the sequence of gaps. A string may be a lattice string both for the scaling ratios and the gaps (and hence, both the zeros and poles of $\zeta_{\mathcal{L}}$ are found by solving a polynomial equation, by Equation (3.1)), but be nonlattice when the scaling ratios and gaps are considered together; that is, the numerator and denominator of $\zeta_{\mathcal{L}}$ have uncommensurable oscillatory periods. 


\section{References}

[BedKS] T. Bedford, M. Keane and C. Series (eds.), Ergodic Theory, Symbolic Dynamics and Hyperbolic Spaces, Oxford Univ. Press, Oxford, 1991.

[BeGo] M. Berger and B. Gostiaux, Differential Geometry: Manifolds, Curves and Surfaces, English translation, Springer-Verlag, Berlin, 1988.

[Ber] M. V. Berry, Distribution of modes in fractal resonators, in: Structural Stability in Physics (W. Güttinger and H. Eikemeier, eds.), Springer-Verlag, Berlin, 1979, pp. 51-53.

[BesT] A. S. Besicovitch and S. J. Taylor, On the complementary intervals of a linear closed set of zero Lebesgue measure, J. London Math. Soc. 29 (1954), 449-459.

[Bo] R. Bowen, Symbolic dynamics for hyperbolic flows, Amer. J. Math. 95 (1973), 429-460.

[BroCa] J. Brossard and R. Carmona, Can one hear the dimension of a fractal?, Commun. Math. Phys. 104 (1986), 103-122.

[Con] A. Connes, Noncommutative Geometry, Academic Press, New York, 1994.

[De] C. Deninger, Evidence for a cohomological approach to analytic number theory, in: Proc. First European Congress of Mathematics (A. Joseph et al., eds.), vol. I, Paris, July 1992, Birkhäuser-Verlag, Basel, 1994, pp. 491-510.

[DoKr] M. M. Dodson and S. Kristensen, Hausdorff dimension and Diophantine approximation, in this volume.

[Fa1] K. J. Falconer, The Geometry of Fractal Sets, Cambridge Univ. Press, Cambridge, 1985.

[Fa2] __ Fractal Geometry: Mathematical foundations and applications, Wiley, Chichester, 1990.

[Fa3] _ On the Minkowski measurability of fractals, Proc. Amer. Math. Soc. 123 (1995), $1115-1124$.

[Fa4] __. Techniques in Fractal Geometry, Wiley, Chichester, 1997.

[Fel] W. Feller, An Introduction to Probability Theory and its Applications, vol. II, Wiley, New York, 1966.

[Fr] M. Frantz, Lacunarity, Minkowski content, and self-similar sets in $\mathbb{R}$, in this volume.

[FreKie] E. Freitag and R. Kiehl, Etale Cohomology and the Weil Conjectures, Springer-Verlag, Berlin, 1988.

[Gat] D. Gatzouras, Lacunarity of self-similar sets and stochastically self-similar sets, Trans. Amer. Math. Soc. 352 (2000), 1953-1983.

[HamLap] B. M. Hambly and M. L. Lapidus, Random fractal strings: their zeta functions, complex dimensions and spectral asymptotics, preprint, 2003.

[Har1] S. Haran, Index theory, potential theory, and the Riemann hypothesis, in: L-functions and Arithmetic, Durham 1990, London Math. Society Lect. Notes Series 153 (1991), 257-270.

[Har2] _ , The Mysteries of the Real Prime, London Mathematical Society Monographs, New Series, vol. 25, Clarendon Press, Oxford, 2001.

[HaWr] G. H. Hardy and E. M. Wright, An Introduction to the Theory of Numbers, fourth edition, Oxford Univ. Press, Oxford, 1960.

[HeLap] C. Q. He and M. L. Lapidus, Generalized Minkowski content, spectrum of fractal drums, fractal strings and the Riemann zeta-function, Memoirs Amer. Math. Soc., No. 608, 127 (1997), 1-97.

[Hub] H. Huber, Zur analytischen Theorie hyperbolischer Raumformen und Bewegungsgruppen, Math. Ann. 138 (1959), 1-26.

[Hut] J. E. Hutchinson, Fractals and self-similarity, Indiana Univ. Math. J. 30 (1981), 713-747.

[In] A. E. Ingham, The Distribution of Prime Numbers, 2nd ed. (reprinted from the 1932 ed.), Cambridge Univ. Press, Cambridge, 1992.

[Kac] M. Kac, Can one hear the shape of a drum?, Amer. Math. Monthly (Slaught Memorial Papers, No. 11) (4) 73 (1966), 1-23.

[Kat] N. M. Katz, An overview of Deligne's proof for varieties over finite fields, Proc. Sympos. Pure Math. 28 (1976), 275-305.

[KigLap1] J. Kigami and M. L. Lapidus, Weyl's problem for the spectral distribution of Laplacians on p.c.f. self-similar fractals, Commun. Math. Phys. 158 (1993), 93-125.

[KigLap2] _ _ Self-similarity of volume measures for Laplacians on p.c.f. self-similar fractals, Commun. Math. Phys. 217 (2001), 165-180.

[Lal1] S. P. Lalley, Packing and covering functions of some self-similar fractals, Indiana Univ. Math. J. 37 (1988), 699-709. 
[Lal2] __ Renewal theorems in symbolic dynamics, with applications to geodesic flows, noneuclidean tessellations and their fractal limits, Acta Math. 163 (1989), 1-55.

[Lap1] M. L. Lapidus, Fractal drum, inverse spectral problems for elliptic operators and a partial resolution of the Weyl-Berry conjecture, Trans. Amer. Math. Soc. 325 (1991), 465-529.

[Lap2] __ Spectral and fractal geometry: From the Weyl-Berry conjecture for the vibrations of fractal drums to the Riemann zeta-function, in: Differential Equations and Mathematical Physics (C. Bennewitz, ed.), Proc. Fourth UAB Internat. Conf. (Birmingham, March 1990), Academic Press, New York, 1992, pp. 151-182.

[Lap3] _ Vibrations of fractal drums, the Riemann hypothesis, waves in fractal media, and the Weyl-Berry conjecture, in: Ordinary and Partial Differential Equations (B. D. Sleeman and R. J. Jarvis, eds.), vol. IV, Proc. Twelfth Internat. Conf. (Dundee, Scotland, UK, June 1992), Pitman Research Notes in Math. Series, vol. 289, Longman Scientific and Technical, London, 1993, pp. 126-209.

[Lap4] __ Analysis on fractals, Laplacians on self-similar sets, noncommutative geometry and spectral dimensions, Topological Methods in Nonlinear Analysis, 4 (1994), 137-195.

[Lap5] __ Fractals and vibrations: Can you hear the shape of a fractal drum?, Fractals 3, No. 4 (1995), 725-736. (Special issue in honor of Benoît B. Mandelbrot's 70th birthday.)

[Lap6] __ Towards a noncommutative fractal geometry? Laplacians and volume measures on fractals, in: Harmonic Analysis and Nonlinear Differential Equations, Contemporary Mathematics, vol. 208, Amer. Math. Soc., 1997, pp. 211-252.

[Lap7] _ _ T-duality, functional equation, and noncommutative spacetime, in: New Interactions of Mathematics with the Natural Sciences and the Humanities (L. Boi, ed.), Springer-Verlag, Berlin, 2003, pp. 1-92.

[Lap8] __, In Search of the Riemann Zeros: Strings, fractal membranes and noncommutative spacetimes, book in preparation; preprint, 2003, 340+(xii)pp.

[LapMa] M. L. Lapidus and H. Maier, The Riemann hypothesis and inverse spectral problems for fractal strings, J. London Math. Soc. (2) 52 (1995), 15-34.

[LapNe] M. L. Lapidus and R. Nest, Fractal membranes and the second quantization of fractal strings, paper in preparation, 2003.

[LapPo1] M. L. Lapidus and C. Pomerance, The Riemann zeta-function and the one-dimensional Weyl-Berry conjecture for fractal drums, Proc. London Math. Soc. (3) 66 (1993), 41-69.

[LapPo2] __ Counterexamples to the modified Weyl-Berry conjecture on fractal drums, Math. Proc. Cambridge Philos. Soc. 119 (1996), 167-178.

[Lap-vF1] M. L. Lapidus and M. van Frankenhuysen, Complex dimensions of fractal strings and oscillatory phenomena in fractal geometry and arithmetic, in: Spectral Problems in Geometry and Arithmetic (T. Branson, ed.), Contemporary Mathematics, vol. 237, Amer. Math. Soc., Providence, R. I., 1999, pp. 87-105.

[Lap-vF2] __ Fractal Geometry and Number Theory (Complex dimensions of fractal strings and zeros of zeta functions), Birkhäuser, Boston, 2000; second revised and enlarged edition expected to appear in 2004.

[Lap-vF3] _ _ A prime orbit theorem for self-similar flows and Diophantine approximation, Contemporary Mathematics 290 (2001), 113-138.

[Lap-vF4] _ Complex dimensions of self-similar fractal strings and Diophantine approximation, J. Experimental Mathematics, No. 1, 42 (2003), 43-69.

[Man] B. B. Mandelbrot, The Fractal Geometry of Nature, revised and enlarged edition, Freeman, San Francisco, 1983.

[Mar] G. Margulis, Certain applications of ergodic theory to the investigation of manifolds of negative curvature, Functional Anal. Appl. 3 (1969), 89-90.

[Mat] P. Mattila, Geometry of Sets and Measures in Euclidean Spaces: Fractals and rectifiability, Cambridge Univ. Press, Cambridge, 1995.

[Mor] P. A. P. Moran, Additive functions of intervals and Hausdorff measure, Math. Proc. Cambridge Philos. Soc. 42 (1946), 15-23.

[ParP1] W. Parry and M. Pollicott, An analogue of the prime number theorem and closed orbits of Axiom A flows, Annals of Math. 118 (1983), 573-591.

[ParP2] _ Z Zeta Functions and the Periodic Orbit Structure of Hyperbolic Dynamics, Astérisque, 187-188, Soc. Math. France, Paris, 1990.

[ParsSh] A. N. Parshin and I. R. Shafarevich (eds.), Number Theory, vols. I \& II, Encyclopedia of Mathematical Sciences, vols. 49 \& 62, Springer-Verlag, Berlin, 1995 \& 1992. 
[Pat] S. J. Patterson, An Introduction to the Theory of the Riemann Zeta-Function, Cambridge Univ. Press, Cambridge, 1988.

[R] D. Ruelle, Generalized zeta-functions for Axiom A basic sets, Bull. Amer. Math. Soc. 82 (1976), 153-156.

[Sch] W. M. Schmidt, Diophantine Approximation, Lecture Notes in Mathematics, 785, SpringerVerlag, Berlin, 1980.

[Si] Y. G. Sinai, The asymptotic behaviour of the number of closed geodesics on a compact manifold of negative curvature, Transl. Amer. Math. Soc. 73 (1968), 227-250.

[St1] R. S. Strichartz, Fourier asymptotics of fractal measures, J. Functional Anal. 89 (1990), $154-187$.

[St2] _ Self-similar measures and their Fourier transforms, I, Indiana Univ. Math. J. 39 (1990), 797-817; II, Trans. Amer. Math. Soc. 336 (1993), 335-361; III, Indiana Univ. Math. J. 42 (1993), 367-411.

[Te] A. Teplyaev, Spectral zeta functions of fractals, preprint, 2003.

[Ti] E. C. Titchmarsh, The Theory of the Riemann Zeta-Function, 2nd ed. (revised by D. R. Heath-Brown), Oxford Univ. Press, Oxford, 1986.

[Wei1] A. Weil, On the Riemann hypothesis in function-fields, Proc. Nat. Acad. Sci. U.S.A. 27 (1941), 345-347. (Reprinted in [Wei4, vol. I, pp. 277-279].)

[Wei2] __, Sur les courbes algébriques et les variétés qui s'en déduisent, Pub. Inst. Math. Strasbourg VII (1948), pp. 1-85. (Reprinted in: Courbes Algébriques et Variétés Abéliennes, Hermann, Paris, 1971.)

[Wei3] A. Weil, Sur les "formules explicites" de la théorie des nombres premiers, Comm. Sém. Math. Lund, Université de Lund, Tome supplémentaire (dédié à Marcel Riesz), (1952), pp. 252-265. (Reprinted in [Wei4, vol. II, pp. 48-61].)

[Wei4] __, André Weil: Oeuvres Scientifiques (Collected Papers), vols. I, II and III, second edition (with corrected printing), Springer-Verlag, Berlin, 1980.

[Wey1] _ _ Das asymptotische Verteilungsgesetz der Eigenwerte linearer partieller Differentialgleichungen, Math. Ann. 71 (1912), 441-479. (Reprinted in [Wey3, vol. I, pp. 393-430].)

[Wey2] __, On the volume of tubes, Amer. J. Math. 61 (1939), 461-472. (Reprinted in [Wey3, vol. III, pp. 658-669].)

[Wey3] __ Hermann Weyl: Gesammelte Abhandlungen (Collected Works), vols. I and III, Springer-Verlag, Berlin, 1968.

Michel L. Lapidus, Department of Mathematics, University of California, RiverSIDE, CA 92521-0135, USA

E-mail address: lapidus@math.ucr.edu

Machiel van Frankenhuijsen, Department of Mathematics, Utah Valley State ColLEGE, OREM, UT 84058-5999, USA

E-mail address: vanframa@uvsc.edu 